\title{
A Multilevel Approach for Analysis and Optimization of Nano-Enhanced Composite Structures
}

\author{
S. DorMohammadi*, M. Rais-Rohani, M. Rouhi
}

\begin{abstract}
A hierarchical framework for multilevel analysis and design of composite material and structural systems is presented. The micro- and macro-level material models are integrated with structural analysis to evaluate the response characteristics of the loaded structure affected by both the nanofiber enhancements and continuous fiber reinforcements in the polymer matrix. Besides the nanofiber waviness, the nanofiber-matrix interphase is also included in evaluation of the homogenized stiffness properties of the matrix. To take advantage of the design features at different length scales, a multilevel optimization approach based on analytical target cascading is developed and applied to material-structural analysis and design optimization of a rectangular composite sandwich plate under in-plane loading conditions. The design variables include the volume fractions of the nanofibers and continuous fibers along with the thicknesses of the core and facesheet plies. Multiple failure modes in the form of global buckling, shear crimping, intracell buckling, and face sheet wrinkling are included as design constraints. Different edge loads are applied to study the effect of loading on optimum design. Besides the significant computational efficiency in the multilevel approach, the analysis detail and the results of the multilevel sandwich plate optimization problem are presented and discussed.
\end{abstract}

\section{Introduction}

Advances in composite materials include the use of nano-inclusions together with conventional reinforcement fibers. Through proper tailoring of the constituent materials, it is possible to enhance both the stiffness and strength characteristics of the resulting multiscale composite by improving the interfacial shear strength (ISS) properties between the conventional fibers and the nano-enhanced matrix. ${ }^{1,2}$ In addition, the overall mechanical properties of the hybrid composite material may be improved through nano-enhancements. ${ }^{3-5}$

Multiscale composite materials and structures offer an opportunity to expand the design space by considering the material properties or structural response attributes at different length scales. The natural hierarchy present in such systems makes them suitable for decomposed optimization approaches. However, given the computational costs associated with the variable fidelity analyses used for function evaluations at different length scales, it is essential to combine the multilevel optimization algorithm with a proper coordination strategy such that the number of expensive function evaluations could be significantly reduced. This is made possible as a result of recent advances in optimization approaches that rely on decomposing a complex system into a hierarchical multilevel framework ${ }^{6-9}$.

Decomposing an optimization problem into a multilevel system results in distribution of design variables and constraints among the individual elements in two or more levels as shown in Fig. 1. A coordination strategy is necessary such that the separately optimized elements satisfy the system-level optimality criteria.

Figure 1. An Example of a hierarchically decomposed multilevel system.

The example problem considered to demonstrate the decomposed analysis and optimization approach is that of a simply-supported rectangular sandwich plate, where the thin-walled honeycomb core is supported by two identical laminated facesheets with generally orthotropic unidirectional layers that consist of continuous fibers and nanofiber-enhanced polymer matrix. The overall hierarchy is shown in

\footnotetext{
${ }^{*}$ Research Scientist, AlphaSTAR Corporation, Long Beach, CA 90804

Tel: +1 562961 7827, E-mail: dsaber@alphastarcorp.com
} 
Fig. 2 with the sandwich plate analysis and design in level 1 at the top, laminate analysis and design in level 2, and enhanced matrix analysis and design in level 3 at the bottom. A micromechanical model is used to calculate the stiffness properties of the nano-enhanced matrix with consideration of the threedimensional interphase that surround the nanofiber inside the matrix as well as the waviness observed in the nanofiber. These features are included in both the analysis and design optimization of the composite sandwich plate as discussed later.

Figure 2. Decomposed hierarchy of nano-enhanced composite sandwich plate.

The remainder of the article is organized as follows: discussion on the optimization approach and the solution strategy, detailed description of the micro- and macro- and structural-level analyses, the optimization problem definition in each element with the discussion of results for the different loading conditions, and summary and conclusions.

\section{Multilevel Design Optimization}

A generic constrained optimization problem in an all-at-once formulation can be expressed as

$$
\begin{array}{lll} 
& \min _{\boldsymbol{x}} f(\boldsymbol{x}) & \\
\text { s.t. } & g_{i}(\boldsymbol{x}) \leq 0 ; & i=1, N_{g} \\
& h_{j}(\boldsymbol{x})=0 ; & j=1, N_{h} \\
& \boldsymbol{x}^{l} \leq \boldsymbol{x} \leq \boldsymbol{x}^{u} &
\end{array}
$$

where $f$ is the objective function, $g_{i}$ is the ith inequality constraint, and $h_{j}$ is the jth equality constraint. The vector of design variables is represented by $\boldsymbol{x}$, with $\boldsymbol{x}^{l}$ and $\boldsymbol{x}^{u}$ as the corresponding vectors of lower and upper bounds, respectively.

By decomposing the problem in Eq. (1) into a multilevel hierarchy, the design variables and design constraints are partitioned among the individual elements. As a result, each element optimization problem is smaller and less complex than the original problem. However, it is necessary to apply a coordination strategy to ensure satisfaction of the system-level targets and optimality criteria in the iterative solution for finding the optimum design.

Analytical target cascading (ATC) ${ }^{6-8}$ provides the framework for the multilevel optimization approach used here. ATC relies on a level-by-level transfer of demands or target variables from above and capabilities or response variables from below between two connected elements in a multilevel system similar to that shown in the right side of Fig. 1. To make the element problems separable, a response variable is introduced for every target variable such that in element $i j$, consistency is ensured using $\mathbf{c}_{i j}=\boldsymbol{t}_{i j}-\boldsymbol{r}_{i j}=0$, where $\boldsymbol{r}_{i j}$ is the vector of responses and $\boldsymbol{t}_{i j}$ is the corresponding vector of known targets.

Various relaxation techniques have been proposed for ATC to augment the objective function in each element with the corresponding consistency constraints between the target and response variables as well as the linking variables that are shared among two or more elements in the same level. Amongst the several formulations suggested in the literature, the augmented Lagrangian formulation based on exponential penalty function $(\mathrm{EPF})^{8,9}$ has been shown to produce better computational efficiency, especially in combination with a single-loop coordination strategy.

By writing the consistency constraints in terms of two inequalities $\left(\boldsymbol{t}_{i j}-\boldsymbol{r}_{i j} \leq 0\right.$ and $\left.\boldsymbol{r}_{i j}-\boldsymbol{t}_{i j} \leq 0\right)$ and using the suggested formulation ${ }^{9}$, the optimization problem $\mathrm{P}_{i j}$ is expressed as 


$$
\begin{aligned}
& \min _{\overline{\boldsymbol{x}}_{i j}} f_{i j}\left(\overline{\boldsymbol{x}}_{i j}\right)+\left\{\frac{\boldsymbol{\mu}_{i j}}{\boldsymbol{a}_{i j}}\left(e^{\boldsymbol{a}_{i j}\left(\boldsymbol{t}_{i j}-\boldsymbol{r}_{i j}\right)}-1\right)+\frac{\boldsymbol{\gamma}_{i j}}{\boldsymbol{b}_{i j}}\left(e^{\boldsymbol{b}_{i j}\left(\boldsymbol{r}_{i j}-\boldsymbol{t}_{i j}\right)}-1\right)\right\} \\
&+\sum_{k \in D_{i j}}\left\{\frac{\boldsymbol{\mu}_{(i+1) k}}{\boldsymbol{a}_{(i+1) k}}\left(e^{\boldsymbol{a}_{(i+1) k}\left(\boldsymbol{t}_{(i+1) k}-\boldsymbol{r}_{(i+1) k}\right)}-1\right)+\frac{\boldsymbol{\gamma}_{(i+1) k}}{\boldsymbol{b}_{(i+1) k}}\left(e^{\boldsymbol{b}_{(i+1) k}\left(\boldsymbol{r}_{(i+1) k}-\boldsymbol{t}_{(i+1) k}\right)}-1\right)\right\}
\end{aligned}
$$

s.t. $\quad \boldsymbol{g}_{i j}\left(\overline{\boldsymbol{x}}_{i j}\right) \leq 0$

$\boldsymbol{h}_{i j}\left(\overline{\boldsymbol{x}}_{i j}\right)=0$

where the vector of local design variables is denoted by $\boldsymbol{x}_{i j}, \boldsymbol{t}_{i j}$ is the vector of target variables shared by element ij and its parent at level i-1, $D_{i j}=\left\{k_{1}, \ldots, k_{D_{i j}}\right\}$ is the set of children of element ij with the local decision variables defined as $\overline{\boldsymbol{x}}_{i j}=\left[\boldsymbol{x}_{i j}, \boldsymbol{r}_{i j}, \boldsymbol{t}_{(i+1) k_{1}}, \ldots, \boldsymbol{t}_{(i+1) k_{n_{i j}}}\right]$. The initial values for multipliers $\boldsymbol{\mu}_{i j}^{0}$ and $\gamma_{i j}^{0}$ are set equal to arbitrary positive numbers and updated using

$\boldsymbol{\mu}_{i j}^{k+1}=\boldsymbol{\mu}_{i j}^{k} \psi_{i j}^{k}=\boldsymbol{\mu}_{i j}^{k} e^{\boldsymbol{a}_{i j}^{k}\left(\boldsymbol{t}_{i j}^{k}-\boldsymbol{r}_{i j}^{k}\right)}$

$\gamma_{i j}^{k+1}=\gamma_{i j}^{k} \bar{\psi}_{i j}^{k}=\gamma_{i j}^{k} e^{\boldsymbol{b}_{i j}^{k}\left(\boldsymbol{r}_{i j}^{k}-\boldsymbol{t}_{i j}^{k}\right)}$

The EPF formulation is combined with a single-loop coordination and updating strategy, where both coordination and parameter updating are performed in a single loop. ${ }^{8}$

Using the preceding decomposition strategy, the multilevel sandwich plate optimization problem follows the arrangement shown in Fig. 3. At the bottom level in element 33, the enhanced matrix properties are optimized based on the neat matrix and nanofiber properties. The local design variable is the volume fraction of the randomly distributed nanofibers given the interphase properties and waviness characteristics, although it is possible to also include the nanofiber properties as design variables. The enhanced matrix properties calculated at this level are treated as input to the mid-level element 22 where the macro-level material modeling and design are performed. The design variables in this level include the thickness of the individual plies in the facesheets as well as the volume fraction of the continuous fibers in each ply. At this level the target value(s) for the bottom level are defined such that together with the local design variables, the facesheet properties are optimized. Similarly, the capabilities at the midlevel are passed up to the top-level element 11 where the overall system requirement are met while minimizing the weight or any other desired performance characteristic of the plate. Additional detail is provided later in discussion of the optimization problem.

Figure 3. Decomposed multilevel optimization framework for the nano-enhanced composite sandwich plate.

Three different coordination schemes (Fig. 4) are considered for solving the three-element problem shown in Fig. 3. In Scheme I, the solution may begin from element 11 or element 33 in a single-loop sequential fashion. In Scheme II, it is necessary for elements 22 and 33 to first reach convergence in each iteration before solving the problem in element 11. Similarly, Scheme III requires convergence between elements 11 and 22 before element 33 is solved. Depending on the analysis and optimization costs in each element, one scheme may be more computationally efficient than the others. Additional detail on solution efficiency is provided later in Section 5.

Figure 4. Alternative coordination strategies for solving the multilevel optimization problem. 


\section{Multilevel Analysis}

Sandwich plates are found in many engineering systems (e.g., automotive and aerospace structures). A sandwich plate typically consists of laminated composite facesheets (with symmetric or unsymmetric stacking sequence) and a cellular core as shown in Fig. 2. Besides the conventional continuous (e.g., carbon) fibers, each unidirectional face-sheet ply is further stiffened by the inclusion of carbon nanofibers (CNFs) mixed with the neat polymer matrix (e.g., vinyl ester). The resulting multiscale composite material in the facesheets can be designed by controlling not only the fiber orientation and thickness of the individual plies but also the properties and composition of the constituent materials (e.g., volume fractions of fibers and CNFs, mean aspect ratio of CNFs).

\subsection{Micro-level material model}

In presence of nano-inclusions, Mori-Tanaka homogenization based on Eshelby's ellipsoidal inclusion model is often used for micromechanical approximation of the stiffness properties such as the Young's modulus and Poisson's ratio. This model assumes weak interaction among the inclusions (inhomogeneities) and perfect interface between inhomogeneity and the surrounding matrix (no interphase). The general ellipsoidal model can be manipulated to produce other inclusion shapes (e.g., elliptic or circular platelets, spherical particles, cylindrical fibers) or voids. Because of agglomeration of the inhomogeneities, Mori-Tanaka-based predictions hold for dilute to semi-dilute matrix-inclusion mixture (i.e., volume fraction below 30\%). Under these assumptions, it is possible to analyze the effect of CNF inclusions on the enhanced matrix properties. For example, using CNF-reinforced vinyl-ester (matrix: $E_{0}=3.5 \mathrm{GPa}, v_{0}=0.3$; nanofiber: $E_{1}=450 \mathrm{GPa}$, and $v_{1}=0.3$ ) with randomly oriented/distributed ellipsoidal CNF, the effect of CNF volume fraction $(f)$ and aspect ratio $(A R)$ on effective modulus $\left(E_{E M}\right)$ of the enhanced matrix are calculated with the corresponding plots shown in Fig. $5 .^{10}$

The saturation point in Fig. 5(a) falls between $A R$ of 100 to 200 for the range of fiber volume fractions considered. Also, Fig. 5(a) shows that the saturated effective modulus nearly doubles for every $20 \%$ increase in the volume fraction. The relationship between $E_{E M}$ and CNF volume fraction in Fig. 5(b) appears to be highly nonlinear. There is minimal change in $E_{E M}$ curve with respect to CNF volume fraction for $\mathrm{AR}>100$. The difference between Mori-Tanaka-based prediction of $E_{E M}$ and that from the rule of mixtures is rather drastic. The effect of nanofibers can be quite significant (e.g., with a $20 \% \mathrm{CNF}$ volume fraction, $E_{E M}$ increases by $600 \%$ ).

Figure 5. Effect of CNF on enhanced matrix properties as a function of (a) aspect ratio and (b) volume fraction.

The effect of three-dimensional inhomogeneous interphase on enhanced matrix properties is modeled using the multi-inclusion (MI) approach ${ }^{12}$ and the procedure presented by Rouhi et al. ${ }^{10}$ and Rouhi ${ }^{11}$. With the interphase treated as a functionally graded (piecewise homogeneous) material, where its effective properties are calculated as ${ }^{12}$

$$
\begin{aligned}
& \boldsymbol{L}^{M I}=\boldsymbol{L}:\left\{\boldsymbol{I}+\sum_{\alpha=1}^{N} f_{\alpha}\left(\boldsymbol{S}^{\Omega}-\boldsymbol{I}\right):\left(\boldsymbol{A}^{\alpha}-\boldsymbol{S}^{\Omega}\right)^{-1}\right\}:\left\{\boldsymbol{I}+\sum_{\alpha=1}^{N} f_{\alpha} \boldsymbol{S}^{\Omega}:\left(\boldsymbol{A}^{\alpha}-\boldsymbol{S}^{\Omega}\right)^{-1}\right\}^{-1} \\
& \boldsymbol{A}^{\alpha}=\left(\boldsymbol{L}-\boldsymbol{L}^{\alpha}\right)^{-1}: \boldsymbol{L}
\end{aligned}
$$

where $\boldsymbol{L}$ is the fourth-order stiffness tensor, $\boldsymbol{I}$ is the fourth-order identity tensor, $f_{\alpha}$ is the volume fraction of the $\alpha$ interphase region, and $S$ is the Eshelby tensor that also depends on the geometry of the multiinclusion model. The curly brackets (braces) in Eq. (4a) represent averaging of the enclosed quantity over all inclusion orientation angles. In Eq. (4a), a multi-inclusion representation consists of an ellipsoid V that 
contains separate phases or nested series of smaller ellipsoids $\Omega_{\alpha}(\alpha=1,2, \ldots, N)$ such that $\Omega_{1} \subset \Omega_{2} \subset \ldots$ $\subset \Omega_{\mathrm{N}} \equiv \mathrm{V}$ is an infinite domain of the matrix material, where the overall elasticity of the multi-phase composite region is treated as a volumetric average of properties of the individual constituent materials.

Through the functionally graded representation of the interphase (Fig. 6), the variation in each elastic property (e.g., Young's modulus, Poisson's ratio) from the surface of the inclusion $(x=0)$ to the outermost layer of the interphase is approximated as

$P=P_{\text {in }}+\left(P_{\text {out }}-P_{\text {in }}\right)\left(\frac{x}{L}\right)^{n} \approx P_{\text {in }}+\left(P_{\text {out }}-P_{\text {in }}\right)\left(\frac{\alpha-1}{N}\right)^{n}$

where $P$ represents an interphase property and $n$ is the interphase variation parameter, with $\alpha$ varying in the range of 1 to $N+1$.

Figure 6. CNF-interphase modeled as multi-inclusion with functionally graded properties.

For example, using CNF-reinforced vinyl-ester (matrix: $E_{0}=3.5 \mathrm{GPa}, v_{0}=0.3$, nanofiber: $E_{1}=450 \mathrm{GPa}$, and $v_{1}=0.3$ ) with randomly oriented/distributed ellipsoidal CNF at $A R=100, f_{1}=0.01$ and assuming a homogeneous interphase $(N=1)$ results in variation of effective modulus $\left(E_{E M}\right)$ as shown in Fig. 7(a). The no interphase case is denoted by No-INP, whereas the other two cases consider an interphase with modulus higher (i.e., High-INP, $E_{I N P}=100 \mathrm{GPa}$ or $\sim 29 E_{0}$ ) or lower (i.e., Low-INP, $E_{I N P}=2 \mathrm{GPa}$ or $\sim 0.6$ $\left.E_{0}\right)$ than that of the neat matrix. ITR represents the ratio of interphase thickness to CNF radius $\left(t_{I N P} / r_{C N F}\right)$. As indicated in Fig. 7(a), for the High-INP case, $E_{E M}$ can improve by as much as $15 \%$ while for the LowINP case, it reduces by $1.4 \%$.

Figure 7. Variation of effective modulus for (a) homogeneous and (b) non-homogeneous interphase.

Using the same CNF-reinforced vinyl-ester material properties but with $f_{1}=0.038$ and treating the interphase as inhomogeneous (piecewise homogeneous) would result in the $E_{E M}$ variation as shown in Fig. 7(b). In this case, the interphase is divided into 20 separate regions $\left(\Omega_{1}\right.$ to $\left.\Omega_{20}\right)$ such that the effective volume fraction of the interphase is kept constant while the interphase variation parameter $n$ is allowed to vary from 0.1 to 5 (see Fig. 6). E-Ratio represents the ratio of moduli in the interphase region 1 to that in region $N=20$, which is assumed to vary from zero to one. ITR is not constant from one case to the other because the intent is to keep the effective volume fraction of the interphase fixed. The results in Fig. 7(b) lead to the conclusion that the overall interphase modulus is more important than its distribution.

The approach proposed by Fisher ${ }^{13}$ is used for modeling the effect of CNF waviness on enhanced matrix properties such that the wavy CNF is assumed to follow a sine wave with specified amplitude and wavelength. The micromechanical analysis is based on the equivalent modulus of straight ellipsoidal CNF calculated using the strain energy formulation and Castigliano's second theorem.

In summary, the CNF material properties, aspect ratio, volume fraction, and waviness together with the interphase model and neat matrix properties are used to calculate the effective modulus of the nanoenhanced matrix for evaluation of ply-level properties.

\subsection{Macro-level material model}

For a lamina with known volume fractions of continuous fibers and nano-enhancement, the selfconsistent field model or the variational bounding method may be used for a more accurate prediction of the transverse modulus. ${ }^{14}$ It is also possible to use the simplified mechanics of materials approach through the rule of mixtures for finding all the in-plane elastic properties (i.e., $E_{1}, E_{2}, G_{12}, v_{12}$ ) of a lamina, where the fiber can be anisotropic but the nano-enhanced matrix is assumed isotropic with homogenized properties. 
With the ply properties of a unidirectional lamina known, the transformed elastic constants at any orientation angle can be found and integrated over the thickness direction to find the macroscopic properties of the resulting laminate. Furthermore, through the application of classical lamination theory and Kirchhoff hypothesis along with Hooke's law and the equilibrium equations, the force-deformation and moment-deformation relationships can be found as

$\left\{\begin{array}{l}\boldsymbol{N} \\ \boldsymbol{M}\end{array}\right\}=\left[\begin{array}{ll}A & B \\ B & D\end{array}\right]\left\{\begin{array}{l}\boldsymbol{\varepsilon} \\ \boldsymbol{\kappa}\end{array}\right\}$

where the in-plane resultant force vector $\boldsymbol{N}$ and moment vector $\boldsymbol{M}$ are related to the mid-plane strains $\boldsymbol{\varepsilon}$ and curvatures $\boldsymbol{\kappa}$ through the extensional, coupling, and bending stiffness matrices (i.e., $A, B, D$ ) found as

$\begin{aligned} A_{i j} & =\sum_{k=1}^{N_{p}} \bar{Q}_{i j}^{k}\left(z_{k}-z_{k-1}\right) \\ B_{i j} & =\frac{1}{2} \sum_{k=1}^{N_{p}} \bar{Q}_{i j}^{k}\left(z_{k}^{2}-z_{k-1}^{2}\right) \\ D_{i j} & =\frac{1}{3} \sum_{k=1}^{N_{p}} \bar{Q}_{i j}^{k}\left(z_{k}^{3}-z_{k-1}^{3}\right)\end{aligned}$

In Eqs. (7), $\bar{Q}_{i j}^{k}$ represents the transformed stiffness matrix of the kth lamina with $z$ measuring the distance through thickness from the mid-plane surface. 


\subsection{Macro-level structural model}

Figure 8 shows the geometric layout and structural parameters of a rectangular sandwich plate with longitudinal and lateral dimensions a and $\mathrm{b}$, ply thickness $t_{i}$ and orientation angle $\theta_{i}$, facesheet thickness $t_{f}$, core cell size $S$, core wall thickness $t_{c}$, and core thickness $h_{c}$.

Figure 8. General layout of the sandwich plate with laminated facesheets and a honeycomb core.

The general small-deflection theory for rectangular orthotropic sandwich plates developed by Libove and Batdorf ${ }^{15}$ and extended by Rao ${ }^{16}$ is used for buckling analysis of simply-supported sandwich plates with anisotropic face sheets. In this theory, curvatures are expressed in terms of the plate's lateral deflection and transverse shear strains in the core. While the facesheets provide most of the bending stiffness, the transverse shear rigidity is governed by the core, which is assumed to have infinite transverse normal rigidity with insignificant in-plane rigidities compared to those of the facesheets. Hence, together with Eq. (6), the transverse shear forces are found as

$\left\{\begin{array}{l}Q_{x} \\ Q_{y}\end{array}\right\}=\left[\begin{array}{cc}S_{x} & 0 \\ 0 & S_{y}\end{array}\right]\left\{\begin{array}{l}\gamma_{x y} \\ \gamma_{y z}\end{array}\right\}$

where transverse shear rigidities are defined as $S_{x}=h_{c} G_{x z}$ and $S_{y}=h_{c} G_{y z}$ with $G_{x z}=(4 / 3)\left(t_{c} / S\right) G_{c}$, $G_{y z}=(8 / 15)\left(t_{c} / S\right) G_{c}$, and $G_{c}$ representing the shear modulus of the core material. The difference in the coefficients of $G_{x z}$ and $G_{y z}$ signifies the effect of core ribbon direction on transverse shear properties in $x z$ and yz planes. According to the theory used, the core's out-of-plane shear moduli contribute to the transverse shear rigidity while only the facesheets contribute to the $A, B$, and $D$ matrices.

The strain energy stored in the facesheets and the core, along with the potential energy associated with the external in-plane reaction forces are calculated for the entire plate. Through the application of Rayleigh-Ritz method and the principle of minimum total potential energy, the resulting eigenvalue problem is solved for the in-plane buckling loads. ${ }^{17,18}$ Once the resultant buckling force components $\left(N_{x_{c r}}, N_{y_{c r}}, N_{x y_{c r}}\right)$ are found, the corresponding buckling stresses are calculated as

$\sigma_{x_{c r}}=\frac{N_{x_{c r}}}{2 t_{f}}$

$\sigma_{y_{c r}}=\frac{N_{y_{c r}}}{2 t_{f}}$

$\tau_{x y_{c r}}=\frac{N_{x y_{c r}}}{2 t_{f}}$

Shear crimping represents the second mode of instability or a degenerate case of plate buckling. This core-dominated instability is caused by excessively low core shear modulus resulting in localized buckling of the core. The shear crimping stress components are found as ${ }^{19,20}$

$$
\begin{aligned}
\sigma_{x_{c r}}^{s c} & =\left(\frac{2}{3}\right) \frac{t_{c} h_{c} G_{c}}{S t_{f}} \\
\sigma_{y_{c r}}^{s c} & =\left(\frac{4}{15}\right) \frac{t_{c} h_{c} G_{c}}{S t_{f}} \\
\tau_{x y_{c r}}^{s c} & =\sqrt{\frac{8}{45}} \frac{t_{c} h_{c} G_{c}}{S t_{f}}
\end{aligned}
$$


The difference in Eqs. (10a) and (10b) is due to the effect of ribbon direction in the honeycomb core. Vinson and Sierakowski ${ }^{20}$ define four parameters to distinguish between the likelihood of global buckling and shear crimping (local instability). Those parameters are considered in this analysis to identify the principal instability mode.

For a sandwich plate with a cellular core, another mode of instability is that associated with intracell buckling, also known as facesheet dimpling. This instability occurs when the facesheet becomes too thin relative to the cell size, causing the local buckling of the facesheet inside the core cell cavity.

$\sigma_{c r}^{i b}=\tau_{c r}^{i b}=\left(\frac{2 \sqrt{\bar{E}_{x} \bar{E}_{y}}}{1-\bar{v}_{x y} \bar{v}_{y x}}\right)\left(\frac{t_{f}}{S}\right)^{2}$

where the in-plane elastic properties denoted as ${ }^{-}$represent the effective laminate properties that are functions of only the $A$ matrix and the facesheet thickness. ${ }^{14}$

Facesheet wrinkling is the last sandwich failure mode considered. This instability occurs when the facesheets are too thin relative to the core. It is similar in form to instability of a thin plate on an elastic foundation, and it is calculated $\mathrm{as}^{20}$

$\sigma_{c r}^{w}=\tau_{c r}^{w}=\sqrt{\frac{16 t_{f} t_{c} E_{c} \sqrt{\bar{E}_{x} \bar{E}_{y}}}{9 h_{c} S\left(1-\bar{v}_{x y} \bar{v}_{y x}\right)}}$

where $E_{c}$ represents the core's Young's modulus in the plate's thickness direction.

\section{Multilevel Sandwich Plate Optimization Problem}

The all-at-once (AAO) sandwich plate optimization problem is defined as

$$
\begin{array}{ll} 
& \min _{x} W_{S P} \\
& g_{c r} \leq 0 ; g_{s c} \leq 0 ; g_{i b} \leq 0 ; g_{w} \leq 0 \\
& 0.0625 \leq S \leq 2.0 \text { in; } 0.0007 \leq t_{c} \leq 0.01 \text { in } \\
& 0.25 \leq V_{f} \leq 0.5 ; 0.006 \leq t_{i} \leq 0.6 \text { in; } 0.1 \leq h_{c} \leq 5.0 \text { in } \\
& 0 \leq V_{C N F} \leq 0.03 \\
& x=\left(V_{C N F}, V_{f}, t_{i}, h_{c}, S, t_{c}\right)
\end{array}
$$

where $W_{S P}$ is the overall weight of the sandwich plate, $S$ is the honeycomb core cell size, $t_{c}$ is the cell wall thickness, $V_{f}$ is the volume fraction of the continuous fibers, $t_{i}$ is the ply thickness, $h_{c}$ is the core thickness, and $V_{C N F}$ is the CNF volume fraction. The design constraints consist of the global buckling $g_{c r}$, shear crimping $g_{s c}$, intracell buckling $g_{i b}$, and facesheet wrinkling $g_{w}$ with the corresponding analyses performed using a sandwich plate design and analysis tool developed through previous research and modified for the structure-material design problem in Fig. 3.

The problem in Eq. (13) is decomposed into a three-level hierarchy as shown in Fig. 3 with microlevel material design at the bottom level, macro-level material design in the middle, and macro-level structural design at the top ${ }^{21}$.

At the micro-level material modeling and design, the target and response variables are chosen as the mechanical properties, i.e., Young's modulus $E_{e m}$ and Poisson's ratio $v_{e m}$ of the nano-enhanced matrix with CNF volume fraction $V_{C N F}$ as the only local design variable. The polymer matrix material is assumed to be vinyl ester thermoset. The element 33 objective function is the inconsistency between the 
enhanced matrix properties and their corresponding target values from the macro-level element above. The element optimization problem based on the EFP formulation is expressed as

$\min _{\bar{x}_{33}}\left\{\frac{\boldsymbol{\lambda}_{33}}{\boldsymbol{a}_{33}}\left(e^{\boldsymbol{a}_{33}\left(\boldsymbol{t}_{33}-\boldsymbol{r}_{33}\right)}-1\right)+\frac{\boldsymbol{\gamma}_{33}}{\boldsymbol{b}_{33}}\left(e^{\boldsymbol{b}_{33}\left(\boldsymbol{r}_{33}-\boldsymbol{t}_{33}\right)}-1\right)\right\}$

s.t. $\quad 0 \leq x_{33} \leq 0.03$

where $\boldsymbol{t}_{33}=\left(E_{e m}^{T}, v_{e m}^{T}\right) ; \boldsymbol{r}_{33}=f_{M-T}\left(x_{33}\right)=\left(E_{e m}^{R}, v_{e m}^{R}\right) ; \bar{x}_{33}=\left(x_{33}\right) ; x_{33}=V_{C N F}$. The superscripts $t$ and $r$ denote target and response, respectively.

At the macro-level material modeling and design, the macroscopic properties of the composite material are of interest, which are affected by both the nano-enhanced matrix and continuous carbon fiber reinforcements. The design focus at this level is mostly on the facesheets' geometric and material properties.

The objective function in element 22 is a combination of the weight of each facesheet $\left(f_{22}\right)$ and the inconsistency between targets and responses associated with both the top and bottom elements in levels 1 and 3, respectively. The targets and responses at this level are the stiffness properties of the facesheets.

The design variables include ply thickness $t_{i}$, Young's modulus and Poisson's ratio of the nano-enhanced matrix $\left(E_{e m}, v_{e m}\right)$, and core thickness $h_{c}$. There are only side constraints in this element problem. Similar to the micro-level material problem, the element optimization problem at the macro-level is formulated as

$$
\begin{aligned}
\min _{\bar{x}_{22}} f_{22}+\left\{\frac{\boldsymbol{\lambda}_{22}}{\boldsymbol{a}_{22}}\left(e^{\boldsymbol{a}_{22}\left(\boldsymbol{t}_{22}-\boldsymbol{r}_{22}\right)}-1\right)+\frac{\boldsymbol{\gamma}_{22}}{\boldsymbol{b}_{22}}\left(e^{\boldsymbol{b}_{22}\left(\boldsymbol{r}_{22}-\boldsymbol{t}_{22}\right)}-1\right)\right\} \\
+\left\{\frac{\boldsymbol{\lambda}_{33}}{\boldsymbol{a}_{33}}\left(e^{\boldsymbol{a}_{33}\left(\boldsymbol{t}_{33}-\boldsymbol{r}_{33}\right)}-1\right)+\frac{\boldsymbol{\gamma}_{33}}{\boldsymbol{b}_{33}}\left(e^{\boldsymbol{b}_{33}\left(\boldsymbol{r}_{33}-\boldsymbol{t}_{33}\right)}-1\right)\right\}
\end{aligned}
$$

s.t. $\quad 0.25 \leq V_{f} \leq 0.5 ; 0.006 \leq t_{i} \leq 0.6$ in; $0.1 \leq h_{c} \leq 5.0$ in

where $f_{22}=\left(\frac{W_{f s}-W_{f s}^{\min }}{W_{f s}^{\max }-W_{f s}^{\min }}\right)$

$$
\begin{aligned}
& \boldsymbol{t}_{22}=\left(\boldsymbol{A}^{T}, \boldsymbol{B}^{T}, \boldsymbol{D}^{T}, t_{f}^{T}, h_{c}^{T}\right) ; \boldsymbol{r}_{22}=\left(\boldsymbol{A}^{R}, \boldsymbol{B}^{R}, \boldsymbol{D}^{R}, t_{f}^{R}, h_{c}^{R}\right) ; \\
& \boldsymbol{A}=f_{A}\left(\boldsymbol{x}_{22}\right) ; \boldsymbol{B}=f_{B}\left(\boldsymbol{x}_{22}\right) ; \boldsymbol{D}=f_{D}\left(\boldsymbol{x}_{22}\right) ; t_{f}=4 \sum t_{i} ; \\
& \boldsymbol{t}_{33}=\left(E_{e m}^{T}, v_{e m}^{T}\right) ; \boldsymbol{r}_{33}=\left(E_{e m}^{R}, v_{e m}^{R}\right) ; \boldsymbol{x}_{22}=\left(V_{f}, t_{i}\right) ; \bar{x}_{22}=\left(h_{c}^{R}, \boldsymbol{t}_{33}, \boldsymbol{x}_{22}\right)
\end{aligned}
$$

The number of layers is assumed to be constant with total plate thickness being $2 t_{f}+h_{c}$. The core material properties $\left(E_{c}, G_{c}, v_{c}\right)$, and panel dimensions are the parameters provided as input to this element problem. A computer code is used to evaluate the macroscopic properties based on the specified inputs.

At the structural-level, the stiffness and strength properties of a rectangular sandwich plate of specified platform dimensions and boundary conditions under a combined in-plane load is optimized. The local design variables for the top-level optimization problem are the honeycomb core cell size $S$ and cell wall thickness $t_{c}$. With the overall weight of the sandwich plate $\left(f_{11}\right)$ together with target-response inconsistencies treated as the objective function, the element 11 optimization problem given as 
$\min _{\bar{x}_{11}} f_{11}+\left\{\frac{\boldsymbol{\lambda}_{22}}{\boldsymbol{a}_{22}}\left(e^{\boldsymbol{a}_{22}\left(\boldsymbol{t}_{22}-\boldsymbol{r}_{22}\right)}-1\right)+\frac{\boldsymbol{\gamma}_{22}}{\boldsymbol{b}_{22}}\left(e^{\boldsymbol{b}_{22}\left(\boldsymbol{r}_{22}-\boldsymbol{t}_{22}\right)}-1\right)\right\}$

s.t. $g_{c r} \leq 0 ; g_{s c} \leq 0 ; g_{i b} \leq 0 ; g_{w} \leq 0 ; 0.0625 \leq S \leq 2.0 \mathrm{in} ; 0.0007 \leq t_{c} \leq 0.01$ in

where

$$
f_{11}=W_{S P} ; \boldsymbol{t}_{22}=\left(\boldsymbol{A}^{T}, \boldsymbol{B}^{T}, \boldsymbol{D}^{T}, t_{f}^{T}, h_{c}^{T}\right) ; \boldsymbol{r}_{22}=\left(\boldsymbol{A}^{R}, \boldsymbol{B}^{R}, \boldsymbol{D}^{R}, t_{f}^{R}, h_{c}^{R}\right) ; \boldsymbol{x}_{11}=\left(S, t_{c}\right) ; \bar{x}_{11}=\left(\boldsymbol{t}_{22}, \boldsymbol{x}_{11}\right)
$$

All the individual computer codes for the material and structural analyses were tested and verified for accuracy prior to their use in solving the multilevel optimization problem.

\section{Results and Discussion}

The simply-supported sandwich plate has 32 plies in each facesheet with a doubly symmetric stacking sequence such that the one-quarter laminate substack has a ply pattern defined as $\theta_{\mathrm{i}}=(0 / 45 /-45 / 90 /$ $90 /-45 / 45 / 0 ; i=1,8$, which is held fixed with the corresponding ply thicknesses treated as design variables. The plate is optimized under four different loading conditions $\left[\mathrm{N}_{x}=-300, \mathrm{~N}_{y}=-300, \mathrm{~N}_{x y}=\right.$ $\left.300, \mathrm{~N}_{x}=\mathrm{N}_{y}=-300 \mathrm{kips} / \mathrm{in}\right]$. The properties used for the various materials are as follows: $\mathrm{CNF}\left(E_{C N F}=\right.$ $\left.65.27 \times 10^{6} \mathrm{psi}, v_{C N F}=0.3\right)$; continuous carbon fiber $\left(E_{f}=27 \times 10^{6} \mathrm{psi}, v_{f}=0.3\right)$; vinyl-ester matrix $\left(E_{m}=0.507 \times 10^{6} \mathrm{psi}, v_{m}=0.3\right)$; and Hexcel 2024 aluminum alloy honeycomb core $\left(E_{c}=10 \times\right.$ $\left.10^{6} \mathrm{psi}, v_{c}=0.3\right)$. The CNF volume fraction $V_{C N F}$ is the design variable at the micro-level to enhance the mechanical properties of the matrix while the continuous fiber volume fraction $V_{f}$ is another variable that appears at the macro-level material design.

As discussed previously, nanofiber waviness and interphase properties could have a significant impact on the properties of the nano-enhanced matrix. Although these properties could be adjusted in the mathematical model, their physical values are subject to considerable uncertainty. For the sake of illustrating the multilevel design framework for composite structures, the following parametric values are assumed: interphase thickness ratio, ITR $=1.0$, interphase variation parameter, $n=1$, nanofiber waviness amplitude $a=0.05 \mu \mathrm{m}$ and wavelength, $\lambda=150 \mu \mathrm{m}$ with aspect ratio $\mathrm{AR}=150$. The values of $I T R$ and $a$ used here are different from those in Ref. [21].

The decomposed multilevel algorithm in Fig. 3 was formulated according to the equations described in the previous sections and implemented in the VisualDOC optimization software with VisualScript managing the communication of information from one element to another. The Modified Method of Feasible Directions (MMFD) in VisualDOC was used to optimize the problems at different levels ${ }^{22}$. MMFD is a gradient-based optimization technique that relies on the solution to a linearized subproblem for finding the usable-feasible search direction followed by a step-size calculation to find an improved design point along the search direction. The iterative procedure is continued until convergence to the point of optimum is achieved. Three principal computer codes of various fidelity are used for calculating the nano-enhanced matrix properties at the micro-level, ply and laminate properties at the macro-level and sandwich plate analysis at the structural-level.

Using an arbitrary set of initial values for the design variables in each element, a double-loop approach $^{8}$ was used to coordinate the iterative solution of the multilevel system with the optimization problem in each element solved using the modified method of feasible directions.

The three coordination strategies in Fig. 4 were analyzed with the results compared to that found using the AAO problem formulation. Given the high computational cost in determining the nano-enhanced matrix properties in element 33, a scheme resulting in the least number of calls to that element would be preferred. In this case, scheme III was found to be the most computationally efficient approach. Whereas the multilevel optimization solution with scheme III took approximately 6 hours $^{\text {a }}$ to complete, the AAO

\footnotetext{
${ }^{a}$ Using a PC with OS: XP SP3; Processor: Intel Core2 Duo CPU E8400 @ 3 GHz and 3.25 GB RAM.
} 
approach took nearly 5 CPU days with a slightly inferior answer. The results shown and discussed in this section are those found using scheme III.

The results in Table 1 are for a square sandwich plate $(\mathrm{a}=\mathrm{b}=12$ in) under uniaxial compression, biaxial compression or in-plane shear with the facesheet material being CNF-enhanced carbon-vinyl ester composite. Given the low effective density of the honeycomb core, the optimum sandwich plate is the one with relatively large core thickness and fairly thin facesheets (11-24\% of core thickness). To eliminate the possibility of intracell buckling given the small facesheet thickness, the core cell size is pushed to its lower bound while the cell wall thickness has reached to or near its upper bound. For all the loading conditions, both CNF and carbon fiber volume fractions are at their upper bounds. The results for the square sandwich plate under uniaxial compression in y-direction show a $68 \%$ increase in weight since the transverse shear stiffness in the xz plane is greater than that in the yz plane. The core is orthotropic due to different properties in the $\mathrm{x}$ (the ribbon direction) and $\mathrm{y}$ (the perpendicular) directions, resulting in different transverse shear stiffnesses in the $\mathrm{xz}$ and yz planes. The thicknesses of the plies with \pm 45 orientation are at their lower bound values for all loading conditions. There is roughly $20 \%$ difference between the core thickness in the case of pure in-plane shear and uniaxial loading in the $\mathrm{x}$ direction, with the overall weight being slightly $(\sim 17 \%)$ higher. It is obvious that the weight of sandwich plate is greater in the case of combined loading than the rest. Both core and facesheet are thicker, resulting in a heavier sandwich plate than in the uniaxial or shear loading case.

Table 1.

The results in Table 2 are for the same square sandwich plate and loading condition as in Table 1, but with the facesheet material made of carbon fiber reinforced vinyl ester composite without any nanoenhancement. As in the case of CNF-enhanced sandwich plates, the facesheets are fairly thin compared to the core thickness (12\% to $25 \%$ ). The core cell size is at its lower bound with the core wall thickness at or near its lower bound. The optimum sandwich plate under uniaxial compression in $\mathrm{x}$ direction is $40 \%$ lighter than that under uniaxial loading in y direction. In all the loading cases, the thicknesses of the \pm 45 plies are at their lower bound values. As in the previous case, the bi-axial compression loading results in the thickest and heaviest plate design.

\section{Table 2.}

Comparing the results in Tables 1 and 2 indicates that utilizing 3\% carbon nanofiber in composite matrix results in approximately $2.0-3.1 \%$ reduction in weight of the sandwich plate. This effect is strongest for the uniaxial compression in the y direction.

Barring the current manufacturing limitations due to the massive increase in viscosity and CNF agglomeration, we considered the possibility of increasing the CNF volume fraction from $3 \%$ to $30 \%$ of the matrix volume. The optimum design for the square sandwich plate with increased nano-enhancement is presented in Table 3. Comparing the results in Tables 1 and 3 indicates that increasing the CNF volume fraction to $30 \%$ results in approximately $2.8-10.0 \%$ reduction in weight of the sandwich plate. It is important to note that nanofiber waviness as well as interphase properties and thickness ratio have a considerable effect on the stiffness properties that can be achieved in the nanofiber enhanced polymer matrix. With greater experimental data, it would be possible to use more realistic values for the aforementioned parameters that could potentially result in much higher impact on the optimum sandwich plate.

Table 3.

The results in Table 4 and 5 are for a rectangular sandwich plate with $a=60$ in. and $b=12$ in. $(a / b=5)$ under various in-plane load cases. Similar to the square plates, the optimum sandwich plates under $\mathrm{y}$ direction loading are around $90 \%$ heavier than the corresponding ones under $\mathrm{x}$ direction loading. 
This difference is higher than that observed for the square sandwich plates in Tables 1 and 2 mainly because a rectangular plate with a large aspect ratio is more susceptible to buckling than a square plate. The 3\% CNF enhancement in composite matrix results in 2.0-3.6\% reduction in weight of the sandwich plate. As in the square plate case, the bi-axial compression causes the optimum weight to be much higher than the other loading cases.

Table 4.

Table 5.

\section{Summary and Conclusions}

A decomposed multi-level approach was introduced for the analysis and optimization of composite structures. The modularity of the multilevel framework faciliates the application of computational models of varying degrees of fidelity and complexity in different elements of the hierarchy. Depending on the computational cost of analysis and optimization in each element of the hierarchy, a different coordination scheme may be used to minimize the overall cost of the optimization problem.

A hybrid composite sandwich plate, in which the facesheets are reinforced by both continuous and nanofibers, was used as an example problem. A modified Mori-Tanaka approach combined with the classical lamination theory and the general small-deflection theory for sandwich plates was adopted in the micro-, macro-, and structural-level analyses, respectively. Through variable-fidelity analyses in different levels, the properties of the nano-enhanced plies were calculated and used in determination of the stiffness properties of a honeycomb core sandwich plate with laminated facesheets. By optimizing the sandwich plate for different in-plane loading conditions and aspect ratios, the effect of nano-enhancement on weight savings and the failure characteristics were investigated while exploring the efficacy of the multilevel optimization approach.

The results of the plate design problem showed that the macro-level geometric properties of the sandwich plate (e.g., ply thickness and orientation angle) have the greatest influence on the overall weight of the structure. Adding nano-reinforcement at the volumetric limit that manufacturing constraints such as agglomeration would allow ( $3 \%$ in this study), may not considerably reduce the weight of the structure (2.0-3.6\% in this study) for two reasons: 1) the buckling load is less sensitive to improving the facesheet properties by adding carbon nanofibers (CNFs), and 2) the very low volume fraction of CNFs would not result in stiffness improvement at such a level that could compensate the size effect and reduce the overall weight by a significant amount. However, as noted earlier, the properties associated with nanofiber waviness and interphase have significant effect on the stiffness properties of the nano-enhanced matrix, and by using less conservative values for these properties, it is possible to see a greater influence of nanoenhancement on the optimum sandwich plate design.

A more significant finding was that the combination of multilevel optimization with a proper coordination strategy could result in substantial reduction in the computational cost in comparison to the all-at-once formulation and solution. This efficiency was due to the drastic difference in the computational time between the micro- and macro-level analyses. Such gains in computational efficiency present an opportunity for future application of the proposed decomposition and optimization framework to more complex multiscale systems involving composite materials.

\section{Acknowledgments}

The partial funding provided for this research by NSF under Grant No. CMMI-0826547 and Raspet Flight Research Laboratory at Mississippi State University is gratefully acknowledged. 


\section{References}

1 Thostenson, E.T., Li, W.Z., Wang, D.Z., Ren, Z.F., and Chou, T.W., "Carbon Nanotube/Carbon Fiber Hybrid Multiscale Composites," Journal of Applied Physics, Vol. 91, No. 9, pp. 6034-6037, 2002.

2 Garg, M., Abdi, F., and McHugh, S., "Analyzing Interlaminar Shear Strength of Multi-Scale Composite Via Combined Finite Element and Progressive Failure Analysis Approach," SAMPE Fall Technical Conference on Multifunctional Materials, Memphis, TN, Sep 2008.

3 Chisholm, N., Mahfuz, H., Rangari, V.K., Ashfaq, A., and Jeelani, S., "Fabrication and Mechanical Characterization of Carbon/SiC-Epoxy Nanocomposites," Composite Structures, Vol. 67, No. 1, pp. 115-124, 2005.

4 Gojny, F.H., Wichmann, M.H.G., Fiedler, B., Bauhofer, W., and Schulte, K., "Influence of Nano-modification on the Mechanical and Electrical Properties of Conventional Fibre-Reinforced Composites," Composites Part A, Vol. 36, No. 11, pp. 1525-1535, 2005.

5 Zhou, Y., Pervin, F., Jeelani, S., and Mallick P.K., "Improvement in Mechanical Properties of Carbon FiberEpoxy Composite Using Carbon Nanofibers," Journal of Material Processing Technology, Vol. 198, No. 1-3, pp. 445-53, 2008.

${ }^{6}$ Kim, M.K., Michelena, N.F., Papalambros, P.Y., Jiang, T., "Target Cascading in Optimal System Design," Journal of Mechanical Design, Vol. 125, No.3, pp. 474-480, 2003.

7 Tosserams, S., Etman, L.F.P., Papalambros, P.Y., Rooda, J.E., "An Augmented Lagrangian Relaxation for Analytical Target Cascading Using the Alternating Directions Method of Multipliers," Structural and Multidisciplinary Optimization, Vol. 31, No. 3, pp. 176-189, $2006 .$.

8 DorMohammadi, S. and Rais-Rohani, M., "Exponential Penalty Function Formulation for Multilevel Optimization Using the Analytical Target Cascading Framework," Structural and Multidisciplinary Optimization, Vol. 47, No. 4, pp. 599-612, 2013.

9 DorMohammadi, S. and Rais-Rohani, M., "Comparison of Alternative Strategies for Multilevel Optimization of Hierarchical Systems," Applied Mathematics, Vol. 3, No. 10A, pp. 1448-1462, 2012.

10 Rouhi, M. and Rais-Rohani, M., "Modeling and probabilistic design optimization of a nanofiber-enhanced composite cylinder for buckling" Composite Structures, Vol. 95, 2013, pp. 346-353.

11 Rouhi, M., Modeling and Optimization of Nano-Enhanced Polymer Composite Structures Under Uncertainty, $\mathrm{PhD}$ Dissertation, Computational Engineering, Mississippi State University, 2011.

12 Nemat-Nasser, S., and Hori, M., Micromechanics: Overall Properties of Heterogeneous Solids, Elsevier, Amsterdam, 1993.

13 Fisher, F., Nanomechanics and Viscoelastic Behavior of Carbon Nanotube Reinforced Polymers, PhD Dissertation, Mechanical Engineering, Northwestern University, 2002.

14 Daniel, I.M. and Ishai, O., Engineering Mechanics of Composite Materials, Oxford University Press, New York, 1994.

15 Libove, C. and Batdorf, S.B., "A General Small-Deflection Theory For Flat Sandwich Plates," NACA TN No. $1526,1948$.

16 Rao, K.M., "Buckling Analysis Of Anisotropic Sandwich Plates Faced With Fiber-Reinforced Plastics," AIAA Journal, Vol. 23, No. 8, 1985, pp. 1247-1253.

17 Rais-Rohani, M. and Marcellier, P., "Buckling and Vibration Analysis of Composite Sandwich Plates with Elastic Rotational Edge Restraints," AIAA Journal, Vol. 37, No. 5, pp. 579-587, 1999.

18 Clements, T., Global-Local Analysis and Design Optimization of a Composite Tilt-Rotor Wing Box Structure, Master's Thesis, Engineering Mechanics, Mississippi State University, 1997.

19 Bruhn, E.F., Analysis and Design of Flight Vehicle Structures, Jacobs publishing, Indianapolis, 1973.

20 Vinson, J.R. and Sierakowski, R.L., The Behavior of Structures Composed of Composite Materials, Marinus Nijhoff, Boston, 1986.

${ }^{21}$ DorMohammadi, S., Rouhi, M., \& Rais-Rohani, M., "Hierarchical Analysis and Optimization of Nano-Enhanced Composite Sandwich Plates," Proceedings of the $55^{\text {th }}$ AIAA/ASME/ASCE/AHS/ASC Structures, Structural Dynamics, and Materials Conference at 2014 AIAA SciTech, National Harbor, MD, Jan 13-17, 2014.

${ }_{22}$ VisualDOC User's manual Version 4.0, Vanderplaats Research and Development, Colorado Springs, Co, 2004. 


\section{Figure Captions}

Figure 1. An Example of a hierarchically decomposed multilevel system.

Figure 2. Decomposed hierarchy of nano-enhanced composite sandwich plate.

Figure 3. Decomposed multilevel optimization framework for the nano-enhanced composite sandwich plate.

Figure 4. Alternative coordination strategies for solving the multilevel optimization problem.

Figure 5. Effect of CNF on enhanced matrix properties as a function of (a) aspect ratio and (b) volume fraction. Figure 6. CNF-interphase modeled as multi-inclusion with functionally graded properties.

Figure 7. Variation of effective modulus for (a) homogeneous and (b) non-homogeneous interphase.

Figure 8. General layout of the sandwich plate with laminated facesheets and a honeycomb core.

\section{Tables}

Table 1. Comparison of optimum designs for $\mathrm{CNF}$ enhanced sandwich plate with $a=b=12 \mathrm{in} .(\mathrm{a} / \mathrm{b}=\mathbf{1})$ under $N_{x}, N_{y}, N_{x y}$, and $N_{x}=N_{y}$ loading.

\begin{tabular}{ccccc}
\hline Design Variables & $\mathrm{N}_{x}$ & $\mathrm{~N}_{y}$ & $\mathrm{~N}_{x y}$ & $\mathrm{~N}_{x}=\mathrm{N}_{y}$ \\
\hline$V_{C N F}$ & 0.03 & 0.03 & 0.03 & 0.03 \\
$V_{f}$ & 0.50 & 0.50 & 0.50 & 0.50 \\
$t_{1}(\theta=0)$, in & 0.0145 & 0.0082 & 0.0176 & 0.0397 \\
$t_{2}(\theta=45)$, in & 0.0060 & 0.0063 & 0.0060 & 0.0060 \\
$t_{3}(\theta=-45)$, in & 0.0060 & 0.0063 & 0.0060 & 0.0060 \\
$t_{4}(\theta=90)$, in & 0.0116 & 0.0366 & 0.0130 & 0.0261 \\
$t_{5}(\theta=90)$, in & 0.0113 & 0.0068 & 0.0169 & 0.0261 \\
$t_{6}(\theta=-45)$, in & 0.0060 & 0.0063 & 0.0060 & 0.0060 \\
$t_{7}(\theta=45)$, in & 0.0060 & 0.0063 & 0.0060 & 0.0060 \\
$t_{8}(\theta=0)$, in & 0.0291 & 0.0510 & 0.0249 & 0.0123 \\
$h_{c}$, in & 1.52 & 3.68 & 1.86 & 4.50 \\
$S$, in & 0.0625 & 0.0625 & 0.0625 & 0.0625 \\
$t_{c}$, in & 0.0100 & 0.0084 & 0.0100 & 0.0100 \\
\hline Weight, lb & 6.46 & 10.82 & 7.31 & 13.34 \\
Total Thickness, in & 2.24 & 4.70 & 2.63 & 5.53 \\
\hline
\end{tabular}


Table 2. Comparison of optimum designs for sandwich plate with $\mathbf{a}=\mathbf{b}=12$ in. $(\mathbf{a} / \mathbf{b}=1)$ under $N_{x}, N_{y}, N_{x y}$, and $N_{x}=N_{y}$ loading.

\begin{tabular}{ccccc}
\hline Design Variables & $\mathrm{N}_{x}$ & $\mathrm{~N}_{y}$ & $\mathrm{~N}_{x y}$ & $\mathrm{~N}_{x}=\mathrm{N}_{y}$ \\
\hline$V_{f}$ & 0.50 & 0.50 & 0.50 & 0.50 \\
$t_{1}(\theta=0)$, in & 0.0198 & 0.0381 & 0.0164 & 0.0233 \\
$t_{2}(\theta=45)$, in & 0.0060 & 0.0060 & 0.0060 & 0.0060 \\
$t_{3}(\theta=-45)$, in & 0.0060 & 0.0060 & 0.0060 & 0.0060 \\
$t_{4}(\theta=90)$, in & 0.0140 & 0.0269 & 0.0216 & 0.0480 \\
$t_{5}(\theta=90)$, in & 0.0136 & 0.0296 & 0.0159 & 0.0104 \\
$t_{6}(\theta=-45)$, in & 0.0060 & 0.0060 & 0.0060 & 0.0060 \\
$t_{7}(\theta=45)$, in & 0.0060 & 0.0060 & 0.0060 & 0.0060 \\
$t_{8}(\theta=0)$, in & 0.0239 & 0.0175 & 0.0235 & 0.0291 \\
$h_{c}$, in & 1.52 & 3.79 & 1.86 & 4.51 \\
$S$, in & 0.0625 & 0.0625 & 0.0625 & 0.0625 \\
$t_{c}$, in & 0.0100 & 0.0082 & 0.0100 & 0.0100 \\
\hline Weight, lb & 6.67 & 11.17 & 7.52 & 13.61 \\
Total Thickness, in & 2.29 & 4.88 & 2.67 & 5.58 \\
\hline
\end{tabular}

Table 3. Comparison of optimum designs for $\mathrm{CNF}$ enhanced sandwich plate with $\mathbf{a}=\mathbf{b}=12$ in. $(\mathrm{a} / \mathbf{b}=\mathbf{1})$ under $N_{x}, N_{y}, N_{x y}$, and $N_{x}=N_{y}$ loading.

\begin{tabular}{ccccc}
\hline Design Variables & $\mathrm{N}_{x}$ & $\mathrm{~N}_{y}$ & $\mathrm{~N}_{x y}$ & $\mathrm{~N}_{x}=\mathrm{N}_{y}$ \\
\hline$V_{C N F}$ & 0.3 & 0.3 & 0.3 & 0.3 \\
$V_{f}$ & 0.50 & 0.50 & 0.50 & 0.50 \\
$t_{1}(\theta=0)$, in & 0.0275 & 0.0114 & 0.0133 & 0.0270 \\
$t_{2}(\theta=45)$, in & 0.0060 & 0.0060 & 0.0060 & 0.0257 \\
$t_{3}(\theta=-45)$, in & 0.0060 & 0.0071 & 0.0060 & 0.0133 \\
$t_{4}(\theta=90)$, in & 0.0108 & 0.0145 & 0.0152 & 0.0139 \\
$t_{5}(\theta=90)$, in & 0.0113 & 0.0265 & 0.0140 & 0.0136 \\
$t_{6}(\theta=-45)$, in & 0.0060 & 0.0071 & 0.0060 & 0.0060 \\
$t_{7}(\theta=45)$, in & 0.0060 & 0.0060 & 0.0060 & 0.0060 \\
$t_{8}(\theta=0)$, in & 0.0126 & 0.0302 & 0.0253 & 0.0198 \\
$h_{c}$, in & 1.51 & 3.11 & 1.86 & 4.50 \\
$S$, in & 0.0625 & 0.0625 & 0.0625 & 0.0625 \\
$t_{c}$, in & 0.0100 & 0.0100 & 0.0100 & 0.0100 \\
\hline Weight, lb & 6.27 & 10.05 & 7.11 & 13.23 \\
Total Thickness, in & 2.20 & 3.98 & 2.59 & 5.51 \\
\hline
\end{tabular}


Table 4. Comparison of optimum designs for CNF enhanced sandwich plate with a = 60 in., $b=12$ in. $(\mathrm{a} / \mathrm{b}=5)$ under $N_{x}, N_{y}, N_{x y}$, and $N_{x}=N_{y}$ loading.

\begin{tabular}{ccccc}
\hline Design Variables & $\mathrm{N}_{x}$ & $\mathrm{~N}_{y}$ & $\mathrm{~N}_{x y}$ & $\mathrm{~N}_{x}=\mathrm{N}_{y}$ \\
\hline$V_{C N F}$ & 0.03 & 0.03 & 0.03 & 0.03 \\
$V_{f}$ & 0.50 & 0.50 & 0.50 & 0.50 \\
$t_{1}(\theta=0)$, in & 0.0111 & 0.0258 & 0.0123 & 0.0286 \\
$t_{2}(\theta=45)$, in & 0.0060 & 0.0060 & 0.0060 & 0.0060 \\
$t_{3}(\theta=-45)$, in & 0.0060 & 0.0060 & 0.0060 & 0.0060 \\
$t_{4}(\theta=90)$, in & 0.0081 & 0.0258 & 0.0105 & 0.0212 \\
$t_{5}(\theta=90)$, in & 0.0176 & 0.0234 & 0.0220 & 0.0310 \\
$t_{6}(\theta=-45)$, in & 0.0060 & 0.0060 & 0.0060 & 0.0060 \\
$t_{7}(\theta=45)$, in & 0.0060 & 0.0060 & 0.0060 & 0.0060 \\
$t_{8}(\theta=0)$, in & 0.0264 & 0.0199 & 0.0229 & 0.0237 \\
$h_{c}$, in & 1.3572 & 3.5653 & 1.6028 & 4.5056 \\
$S$, in & 0.0625 & 0.0625 & 0.0625 & 0.0625 \\
$t_{c}$, in & 0.0100 & 0.0100 & 0.0100 & 0.0100 \\
\hline Weight, lb & 30.15 & 56.40 & 33.30 & 66.75 \\
Total Thickness, in & 2.05 & 4.52 & 2.34 & 5.53 \\
\hline
\end{tabular}

Table 5. Comparison of optimum designs for sandwich plate with $a=60 \mathrm{in} ., \mathrm{b}=12 \mathrm{in} .(\mathrm{a} / \mathrm{b}=5)$ under $N_{x}, N_{y}, N_{x y}$, and $N_{x}=N_{y}$ loading.

\begin{tabular}{ccccc}
\hline Design Variables & $\mathrm{N}_{x}$ & $\mathrm{~N}_{y}$ & $\mathrm{~N}_{x y}$ & $\mathrm{~N}_{x}=\mathrm{N}_{y}$ \\
\hline$V_{f}$ & 0.50 & 0.50 & 0.50 & 0.50 \\
$t_{1}(\theta=0)$, in & 0.0154 & 0.0222 & 0.0182 & 0.0314 \\
$t_{2}(\theta=45)$, in & 0.0060 & 0.0060 & 0.0060 & 0.0060 \\
$t_{3}(\theta=-45)$, in & 0.0060 & 0.0060 & 0.0060 & 0.0060 \\
$t_{4}(\theta=90)$, i & 0.0078 & 0.0335 & 0.0170 & 0.0128 \\
$t_{5}(\theta=90)$, in & 0.0242 & 0.0201 & 0.0201 & 0.0411 \\
$t_{6}(\theta=-45)$, in & 0.0060 & 0.0060 & 0.0060 & 0.0060 \\
$t_{7}(\theta=45)$, in & 0.0060 & 0.0060 & 0.0060 & 0.0060 \\
$t_{8}(\theta=0)$, in & 0.0204 & 0.0250 & 0.0173 & 0.0254 \\
$h_{c}$, in & 1.3737 & 3.5653 & 1.6123 & 4.5075 \\
$S$, in & 0.0625 & 0.0625 & 0.0625 & 0.0625 \\
$t_{c}$, in & 0.0100 & 0.0100 & 0.0100 & 0.0100 \\
\hline Weight, lb & 31.28 & 57.63 & 34.39 & 68.08 \\
Total Thickness, in & 2.11 & 4.56 & 2.39 & 5.59 \\
\hline
\end{tabular}




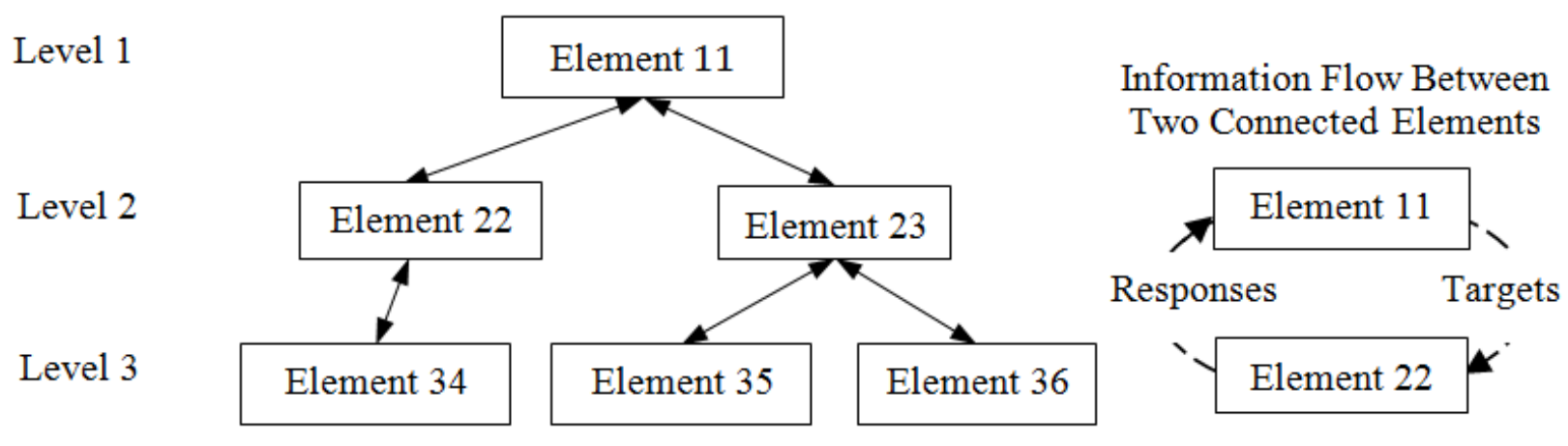

Figure 1 


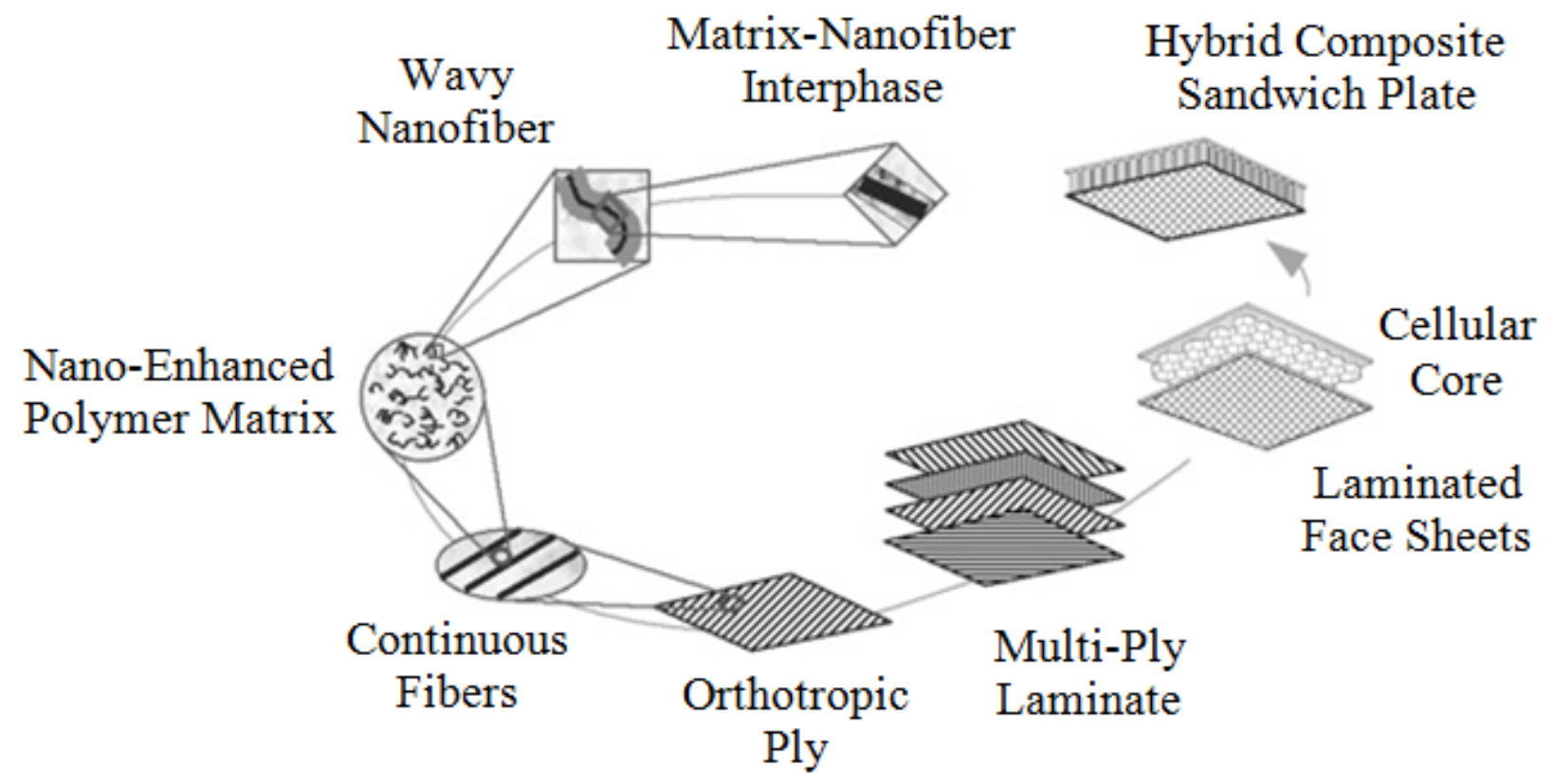

Figure 2 


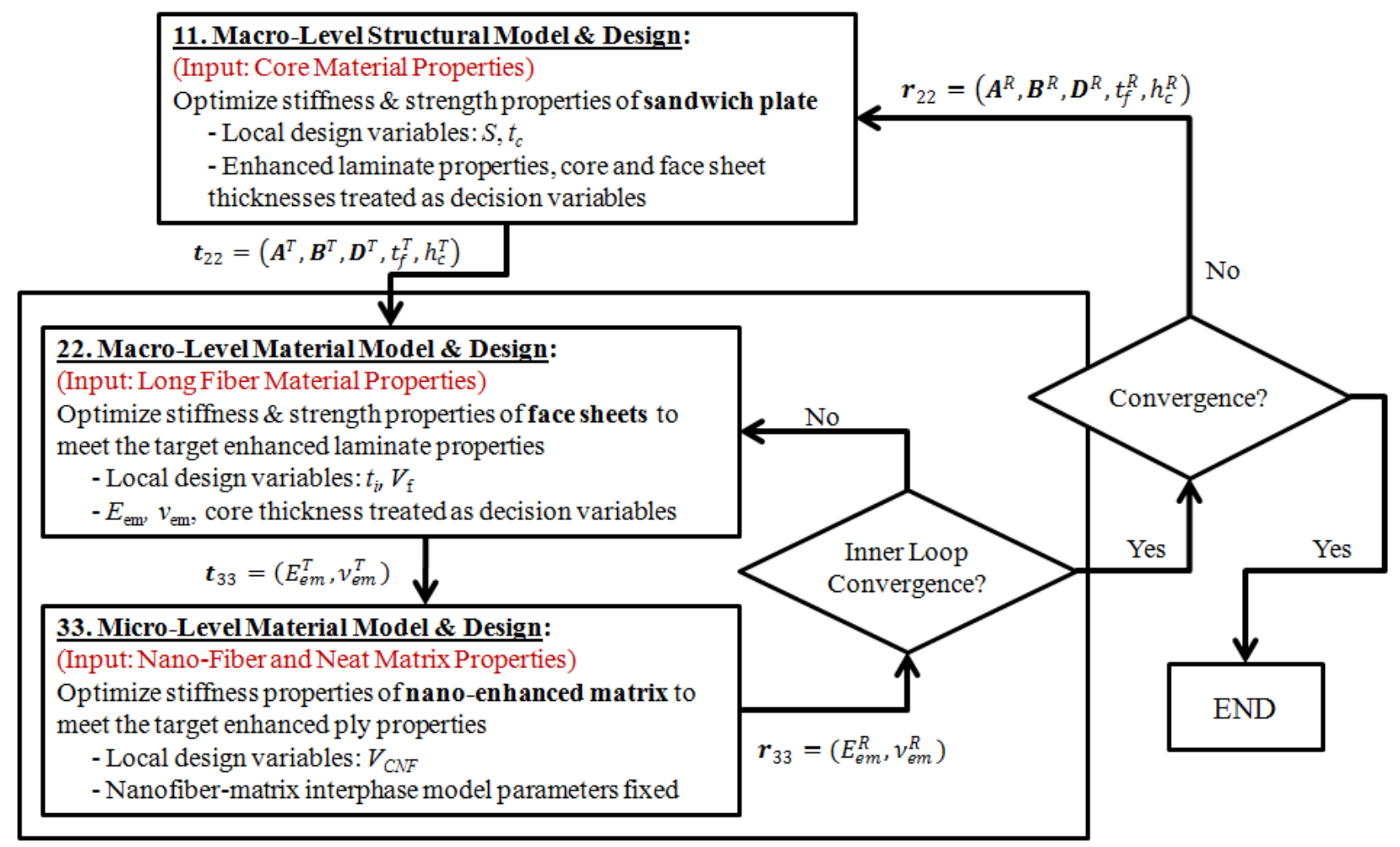

Figure 3 


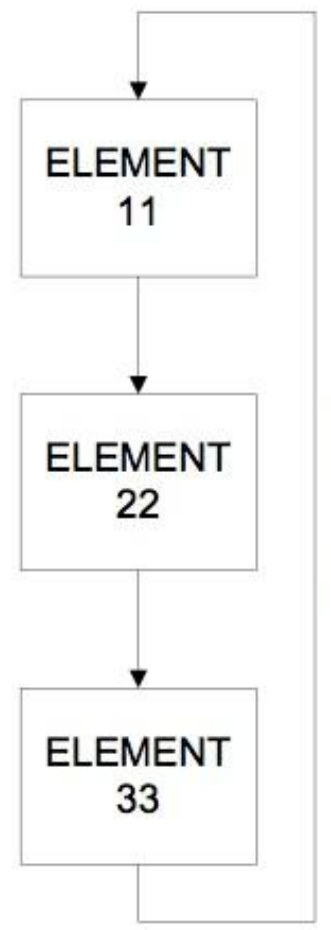

Scheme I

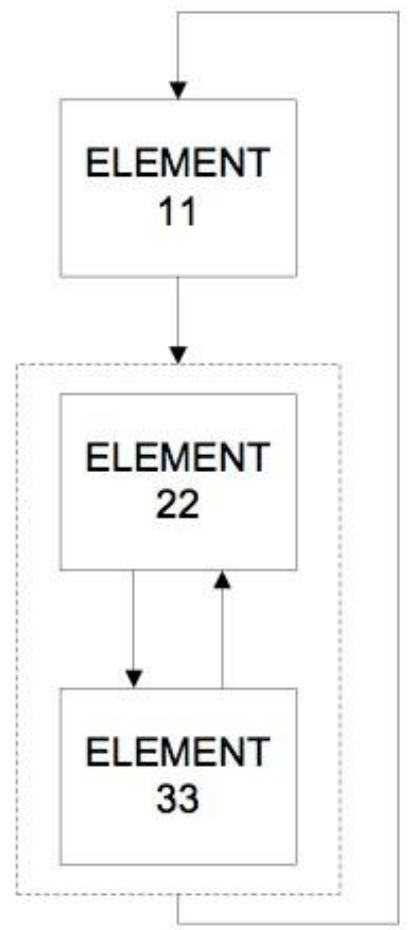

Scheme II

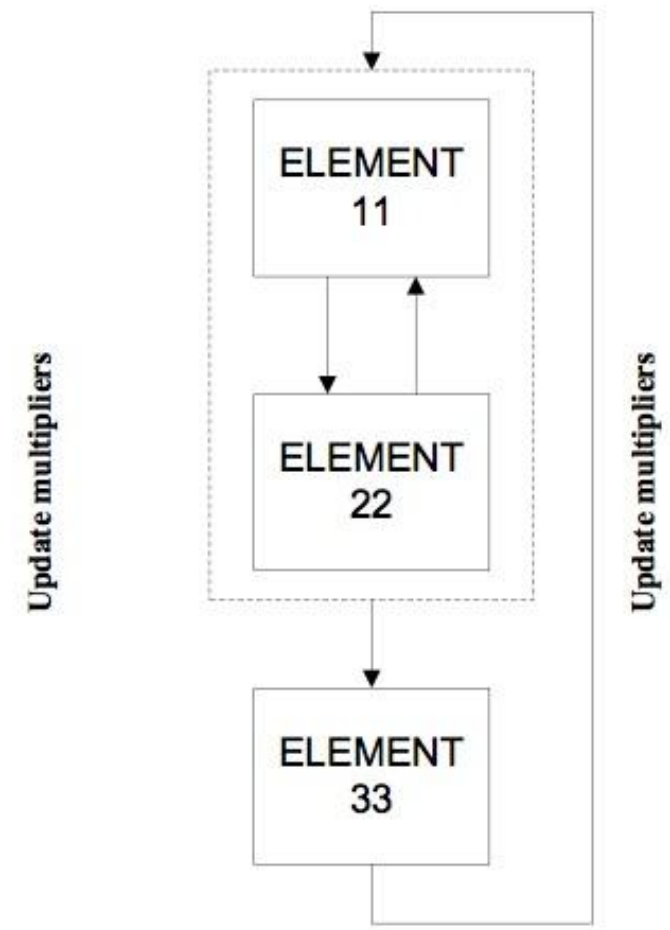

Scheme III

Figure 4 

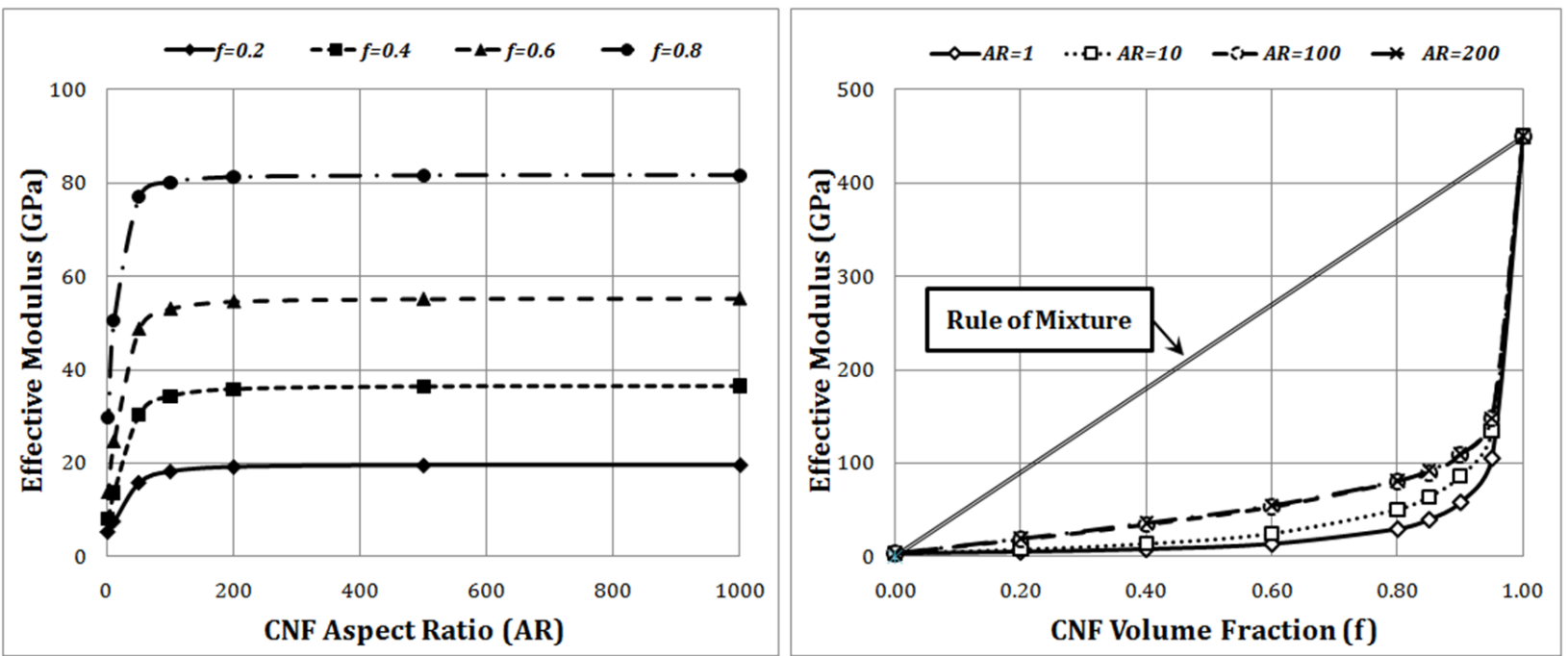

Figure 5 
Nano-Enhanced

Polymer Matrix

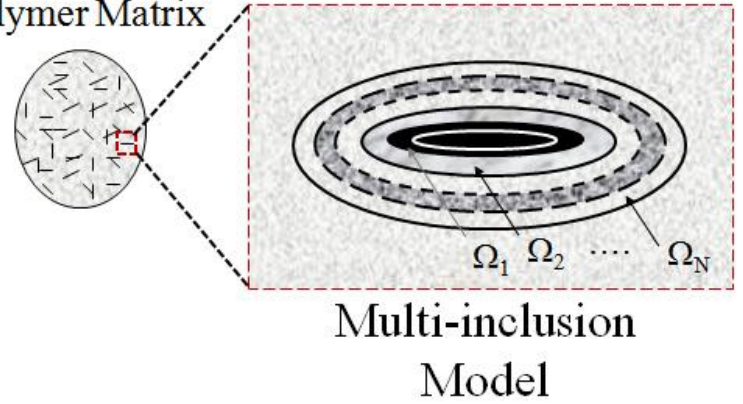

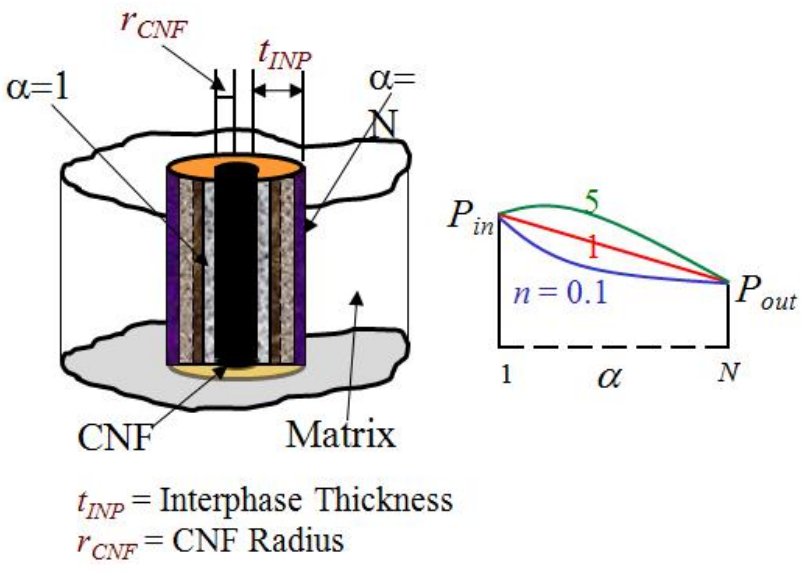

Figure 6 

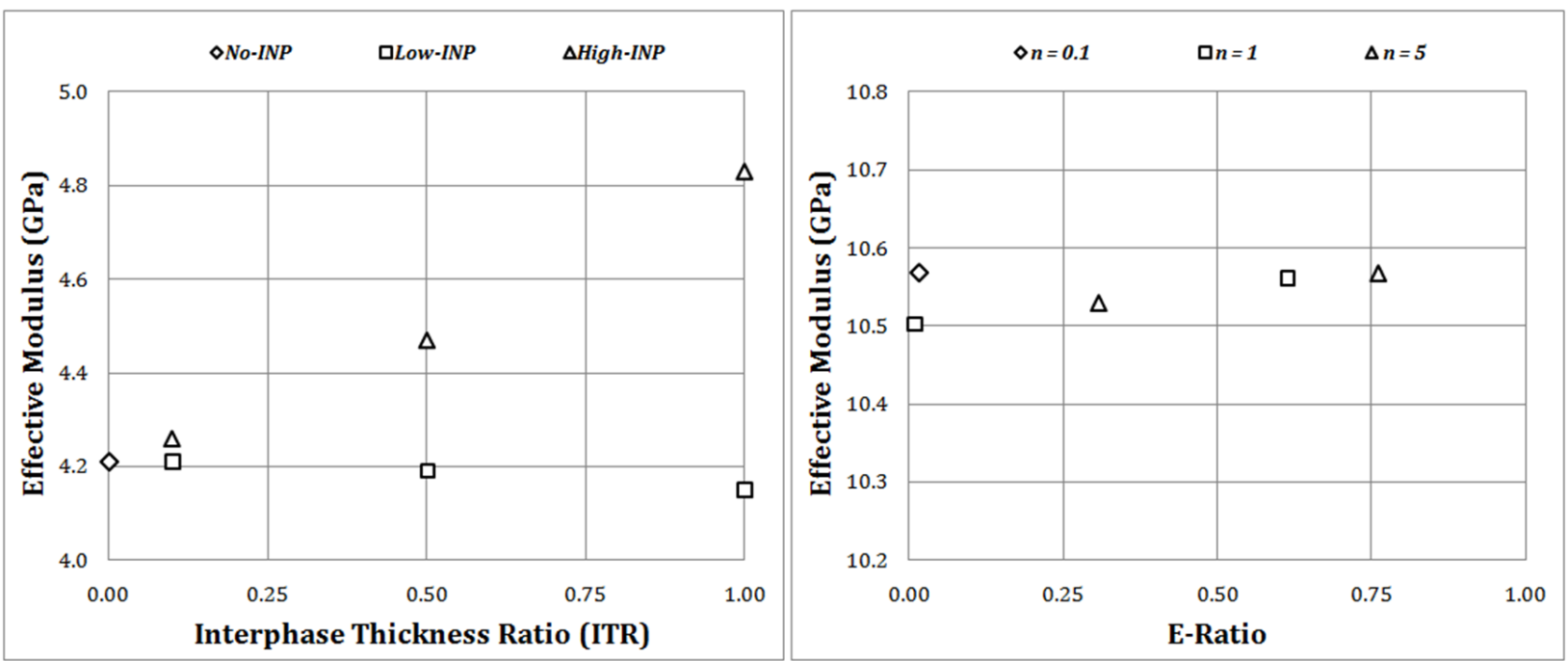

Figure 7 

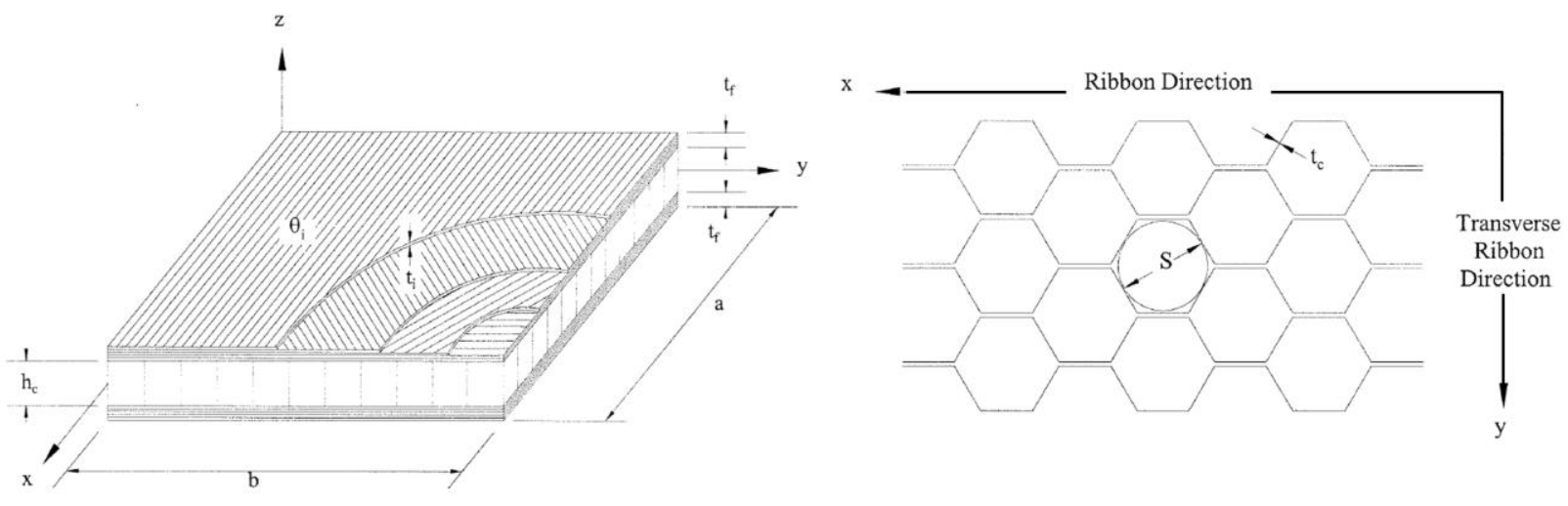

Figure 8 